\title{
Implicit nonlinear fractional differential equations of variable order
}

\author{
Amar Benkerrouche ${ }^{1}$, Mohammed Said Souid ${ }^{2}$, Kanokwan Sitthithakerngkiet ${ }^{3 *}$ (DD and Ali Hakem ${ }^{1}$
}

\author{
"Correspondence: \\ kanokwan.s@sci.kmutnb.ac.th \\ ${ }^{3}$ Intelligent and Nonlinear Dynamic \\ Innovations Research Center, \\ Department of Mathematics, \\ Faculty of Applied Science, King \\ Mongkut's University of Technology \\ North Bangkok (KMUTNB), \\ Wongsawang, Bangsue, 10800, \\ Bangkok, Thailand \\ Full list of author information is \\ available at the end of the article
}

\begin{abstract}
In this manuscript, we examine both the existence and the stability of solutions to the implicit boundary value problem of Caputo fractional differential equations of variable order. We construct an example to illustrate the validity of the observed results.
\end{abstract}

MSC: 26A33; 34K37

Keywords: Fractional differential equations of variable order; Boundary value problem; Fixed point theorem; Ulam-Hyers stability

\section{Introduction}

The idea of fractional calculus is to replace the natural numbers in the derivative's order with rational ones. Although it seems an elementary consideration, it has an exciting relevance explaining some physical phenomena. Especially in the last two decades, significant numbers of papers appeared on this topic, some papers deal with the existence of solutions to problems of variable order; see e.g. [3, 4, 9, 10, 12].

In particular, [2] Benchohra et al. studied the existence and uniqueness results for the following nonlinear implicit fractional differential equations:

$$
\left\{\begin{array}{l}
{ }^{c} D_{0^{+}}^{u} x(t)=f\left(t, x(t),{ }^{c} D_{0^{+}}^{u} x(t)\right), \quad t \in[0, T], 0<T<+\infty, 1<u \leq 2, \\
x(0)=x_{0}, \quad x(T)=x_{1},
\end{array}\right.
$$

where $f$ is a given function, $x_{0}, x_{1} \in \Re$, and ${ }^{c} D_{0^{+}}^{u}$ is the Caputo fractional derivative of order $u$.

Inspired by [2] and $[3,4,9,10,12]$, we deal with the boundary value problem (BVP)

$$
\left\{\begin{array}{l}
{ }^{c} D_{0^{+}}^{u(t)} x(t)=f_{1}\left(t, x(t),{ }^{c} D_{0^{+}}^{u(t)} x(t)\right), \quad t \in J:=[0, T] \\
x(0)=0, \quad x(T)=0,
\end{array}\right.
$$

where $u: J \rightarrow(1,2], f_{1}: J \times \Re \times \Re \rightarrow \Re$ is a continuous function and ${ }^{c} D_{0^{+}}^{u(t)}$ is the Caputo fractional derivative of variable-order $u(t)$.

In this paper, we shall look for a solution of (1). Further, we study the stability of the obtained solution of (1) in the sense of Ulam-Hyers $(U H)$.

C) The Author(s) 2021. This article is licensed under a Creative Commons Attribution 4.0 International License, which permits use, sharing, adaptation, distribution and reproduction in any medium or format, as long as you give appropriate credit to the original author(s) and the source, provide a link to the Creative Commons licence, and indicate if changes were made. The images or other third party material in this article are included in the article's Creative Commons licence, unless indicated otherwise in a credit line to the material. If material is not included in the article's Creative Commons licence and your intended use is not permitted by statutory regulation or exceeds the permitted use, you will need to obtain permission directly from the copyright holder. To view a copy of this licence, visit http://creativecommons.org/licenses/by/4.0/. 


\section{Preliminaries}

This section introduces some important fundamental definitions that will be needed for obtaining our results in the next sections.

The symbol $C(J, \Re)$ represents the Banach space of continuous functions $x: J \rightarrow \Re$ with the norm

$$
\|x\|=\operatorname{Sup}\{|x(t)|: t \in J\}
$$

For $-\infty<a_{1}<a_{2}<+\infty$, we consider the mappings $u(t):\left[a_{1}, a_{2}\right] \rightarrow(0,+\infty)$ and $v(t)$ : $\left[a_{1}, a_{2}\right] \rightarrow(n-1, n), n \in N$. Then the left Caputo fractional integral (CFI) of variable-order $u(t)$ for the function $f_{2}(t)[7,8,11]$ is

$$
I_{a_{1}^{+}}^{u(t)} f_{2}(t)=\int_{a_{1}}^{t} \frac{(t-s)^{u(t)-1}}{\Gamma(u(t))} f_{2}(s) d s, \quad t>a_{1}
$$

and the left Caputo fractional derivative (CFD) of variable-order $v(t)$ for the function $f_{2}(t)$ $[7,8,11]$ is

$$
{ }^{c} D_{a_{1}^{+}}^{v(t)} f_{2}(t)=\int_{a_{1}}^{t} \frac{(t-s)^{n-v(t)-1}}{\Gamma(n-v(t))} f_{2}^{(n)}(s) d s, \quad t>a_{1} .
$$

As anticipated, in the case of $u(t)$ and $v(t)$ being constant, then CFI and CFD coincide with the standard Caputo fractional derivative and integral, respectively; see e.g. [6-8].

Recall the following pivotal observation.

Lemma 2.1 ([6]) Let $\alpha_{1}, \alpha_{2}>0, a_{1}>0, f_{2} \in L\left(a_{1}, a_{2}\right),{ }^{c} D_{a_{1}^{+}}^{\alpha_{1}} f_{2} \in L\left(a_{1}, a_{2}\right)$. Then the differential equation

$$
{ }^{c} D_{a_{1}^{+}}^{\alpha_{1}} f_{2}=0
$$

has the unique solution

$$
f_{2}(t)=\omega_{0}+\omega_{1}\left(t-a_{1}\right)+\omega_{2}\left(t-a_{1}\right)^{2}+\cdots+\omega_{n-1}\left(t-a_{1}\right)^{n-1}
$$

and

$$
I_{a_{1}^{+}}^{\alpha_{1} c} D_{a_{1}^{+}}^{\alpha_{1}} f_{2}(t)=f_{2}(t)+\omega_{0}+\omega_{1}\left(t-a_{1}\right)+\omega_{2}\left(t-a_{1}\right)^{2}+\cdots+\omega_{n-1}\left(t-a_{1}\right)^{n-1}
$$

with $n-1<\alpha_{1} \leq n, \omega_{\ell} \in \Re, \ell=0,1, \ldots, n-1$.

Furthermore,

$$
{ }^{c} D_{a_{1}^{+}}^{\alpha_{1}} I_{a_{1}^{+}}^{\alpha_{1}} f_{2}(t)=f_{2}(t)
$$

and

$$
I_{a_{1}^{\prime}}^{\alpha_{1}} I_{a_{1}^{+}}^{\alpha_{2}} f_{2}(t)=I_{a_{1}^{+}}^{\alpha_{2}} I_{a_{1}^{+}}^{\alpha_{1}} f_{2}(t)=I_{a_{1}^{+}}^{\alpha_{1}+\alpha_{2}} f_{2}(t)
$$


Remark $2.1([13,15,16])$ Note that the semigroup property is not fulfilled for general functions $u(t), v(t)$, i.e.,

$$
I_{a_{1}^{+}}^{u(t)} I_{a_{1}^{+}}^{v(t)} f_{2}(t) \neq I_{a_{1}^{+}}^{u(t)+v(t)} f_{2}(t)
$$

Example 2.1 Let

$$
\begin{aligned}
u(t)=t, \quad t & \in[0,4], \quad v(t)=\left\{\begin{array}{ll}
2, & t \in[0,1], \\
3, & t \in] 1,4],
\end{array} \quad f_{2}(t)=2, \quad t \in[0,4],\right. \\
I_{0^{+}}^{u(t)} I_{0^{+}}^{v(t)} f_{2}(t) & =\int_{0}^{t} \frac{(t-s)^{u(t)-1}}{\Gamma(u(t))} \int_{0}^{s} \frac{(s-\tau)^{v(s)-1}}{\Gamma(v(s))} f_{2}(\tau) d \tau d s \\
& =\int_{0}^{t} \frac{(t-s)^{t-1}}{\Gamma(t)}\left[\int_{0}^{1} \frac{(s-\tau)}{\Gamma(2)} 2 d \tau+\int_{1}^{s} \frac{(s-\tau)^{2}}{\Gamma(3)} 2 d \tau\right] d s \\
& =\int_{0}^{t} \frac{(t-s)^{t-1}}{\Gamma(t)}\left[2 s-1+\frac{(s-1)^{3}}{3}\right] d s,
\end{aligned}
$$

and

$$
I_{0^{+}}^{u(t)+v(t)} f_{2}(t)=\int_{0}^{t} \frac{(t-s)^{u(t)+v(t)-1}}{\Gamma(u(t)+v(t))} f_{2}(s) d s
$$

So, we get

$$
\begin{aligned}
\left.I_{0^{+}}^{u(t)} I_{0^{+}}^{v(t)} f_{2}(t)\right|_{t=3}= & \int_{0}^{3} \frac{(3-s)^{2}}{\Gamma(3)}\left[2 s-1+\frac{(s-1)^{3}}{3}\right] d s \\
= & \frac{21}{10}, \\
\left.I_{0^{+}}^{u(t)+v(t)} f_{2}(t)\right|_{t=3}= & \int_{0}^{3} \frac{(3-s)^{u(t)+v(t)-1}}{\Gamma(u(t)+v(t))} f_{2}(s) d s \\
= & \int_{0}^{1} \frac{(3-s)^{4}}{\Gamma(5)} 2 d s+\int_{1}^{3} \frac{(3-s)^{5}}{\Gamma(6)} 2 d s \\
= & \frac{1}{12} \int_{0}^{1}\left(s^{4}-12 s^{3}+54 s^{2}-108 s+81\right) d s \\
& +\frac{1}{60} \int_{1}^{3}\left(-s^{5}+15 s^{4}-90 s^{3}+270 s^{2}-405 s+243\right) d s \\
= & \frac{665}{180} .
\end{aligned}
$$

Therefore, we obtain

$$
\left.I_{0^{+}}^{u(t)} I_{0^{+}}^{v(t)} f_{2}(t)\right|_{t=3} \neq\left. I_{0^{+}}^{u(t)+v(t)} f_{2}(t)\right|_{t=3} .
$$

Lemma 2.2 ([18]) Let $u: J \rightarrow(1,2]$ be a continuous function, then, for $f_{2} \in C_{\delta}(J, \Re)=$ $\left\{f_{2}(t) \in C(J, \Re), t^{\delta} f_{2}(t) \in C(J, \Re), 0 \leq \delta \leq 1\right\}$, the variable order fractional integral $I_{0^{+}}^{u(t)} f_{2}(t)$ exists for any points on $J$. 
Lemma 2.3 ([18]) Let $u: J \rightarrow(1,2]$ be a continuous function, then $I_{0^{+}}^{u(t)} f_{2}(t) \in C(J, \Re)$ for $f_{2} \in C(J, \Re)$.

Definition 2.1 $([5,14,17])$ Let $I \subset \Re, I$ is called a generalized interval if it is either an interval, or $\left\{a_{1}\right\}$ or $\emptyset$.

A finite set $\mathcal{P}$ is called a partition of $I$ if each $x$ in $I$ lies in exactly one of the generalized intervals $E$ in $\mathcal{P}$.

A function $g: I \rightarrow \Re$ is called piecewise constant with respect to partition $\mathcal{P}$ of $I$ if for any $E \in \mathcal{P}, g$ is constant on $E$.

Theorem 2.1 (Krasnoselskii fixed point theorem [6]) Let $S$ be a closed, bounded and convex subset of a real Banach space $E$ and let $W_{1}$ and $W_{2}$ be operators on $S$ satisfying the following conditions:

(i) $W_{1}(S)+W_{2}(S) \subset S$,

(ii) $W_{1}$ is continuous on $S$ and $W_{1}(S)$ is a relatively compact subset of $E$,

(iii) $W_{2}$ is a strict contraction on $S$, i.e., there exists $k \in[0,1)$, such that

$$
\left\|W_{2}(x)-W_{2}(y)\right\| \leq k\|x-y\|
$$

for every $x, y \in S$.

Then there exists $x \in S$ such that $W_{1}(x)+W_{2}(x)=x$.

Definition 2.2 ([1]) Equation (1) is $(U H)$ stable if there exists $c_{f_{1}}>0$, such that, for any $\epsilon>0$ and for every solution $z \in C(J, \Re)$ of the following inequality:

$$
\left|{ }^{c} D_{0^{+}}^{u(t)} z(t)-f_{1}\left(t, z(t),{ }^{c} D_{0^{+}}^{u(t)} z(t)\right)\right| \leq \epsilon, \quad t \in J
$$

there exists a solution $x \in C(J, \Re)$ of Eq. (1) with

$$
|z(t)-x(t)| \leq c_{f_{1}} \epsilon, \quad t \in J
$$

\section{Existence of solutions}

Let us introduce the following assumption:

(H1) Let $n \in N$ be an integer, $\mathcal{P}=\left\{J_{1}:=\left[0, T_{1}\right], J_{2}:=\left(T_{1}, T_{2}\right], J_{3}:=\left(T_{2}, T_{3}\right], \ldots, J_{n}:=\right.$ $\left.\left(T_{n-1}, T\right]\right\}$ be a partition of the interval $J$, and let $u(t): J \rightarrow(1,2]$ be a piecewise constant function with respect to $\mathcal{P}$, i.e.,

$$
u(t)=\sum_{\ell=1}^{n} u_{\ell} I_{\ell}(t)= \begin{cases}u_{1}, & \text { if } t \in J_{1}, \\ u_{2} & \text { if } t \in J_{2}, \\ \vdots & \\ u_{n} & \text { if } t \in J_{n},\end{cases}
$$

where $1<u_{\ell} \leq 2$ are constants, and $I_{\ell}$ is the indicator of the interval $J_{\ell}:=\left(T_{\ell-1}, T_{\ell}\right], \ell=$ $1,2, \ldots, n$, (with $T_{0}=0, T_{n}=T$ ) such that

$$
I_{\ell}(t)= \begin{cases}1 & \text { for } t \in J_{\ell} \\ 0 & \text { for elsewhere }\end{cases}
$$


For each $\ell \in\{1,2, \ldots, n\}$, the symbol $E_{\ell}=C\left(J_{\ell}, \Re\right)$, indicates the Banach space of continuous functions $x: J_{\ell} \rightarrow \Re$ equipped with the norm

$$
\|x\|_{E_{\ell}}=\sup _{t \in J_{\ell}}|x(t)|
$$

Then, for any $t \in J_{\ell}, \ell=1,2, \ldots, n$, the left Caputo fractional derivative of variable order $u(t)$ for the function $x(t) \in C(J, \Re)$, defined by (3), could be presented as a sum of left Caputo fractional derivatives of constant-orders $u_{\ell}, \ell=1,2, \ldots, n$

$$
{ }^{c} D_{0^{+}}^{u(t)} x(t)=\int_{0}^{T_{1}} \frac{(t-s)^{1-u_{1}}}{\Gamma\left(2-u_{1}\right)} x^{(2)}(s) d s+\cdots+\int_{T_{\ell-1}}^{t} \frac{(t-s)^{1-u_{\ell}}}{\Gamma\left(2-u_{\ell}\right)} x^{(2)}(s) d s .
$$

Thus, according to (5), the BVP (1) can be written for any $t \in J_{\ell}, \ell=1,2, \ldots, n$ in the form

$$
\int_{0}^{T_{1}} \frac{(t-s)^{1-u_{1}}}{\Gamma\left(2-u_{1}\right)} x^{(2)}(s) d s+\cdots+\int_{T_{\ell-1}}^{t} \frac{(t-s)^{1-u_{\ell}}}{\Gamma\left(2-u_{\ell}\right)} x^{(2)}(s) d s=f_{1}\left(t, x(t),{ }^{c} D_{0^{+}}^{u(t)} x(t)\right)
$$

In what follows we shall introduce the solution to the BVP (1).

Definition 3.1 The BVP (1) has a solution, if there are functions $x_{\ell}, \ell=1,2, \ldots, n$, so that $x_{\ell} \in C\left(\left[0, T_{\ell}\right], \Re\right)$, fulfilling Eq. (6), and $x_{\ell}(0)=0=x_{\ell}\left(T_{\ell}\right)$.

Let the function $x \in C(J, \Re)$ be such that $x(t) \equiv 0$ on $t \in\left[0, T_{\ell-1}\right]$ and such that it solves the integral equation (6). Then (6) is reduced to

$$
{ }^{c} D_{T_{\ell-1}^{+}}^{u_{\ell}} x(t)=f_{1}\left(t, x(t),{ }^{c} D_{T_{\ell-1}^{+}}^{u_{\ell}} x(t)\right), \quad t \in J_{\ell} .
$$

We shall deal with the following BVP:

$$
\left\{\begin{array}{l}
{ }^{c} D_{T_{\ell-1}^{+}}^{u_{\ell}} x(t)=f_{1}\left(t, x(t),{ }^{c} D_{T_{\ell-1}^{+}}^{u_{\ell}} x(t)\right), \quad t \in J_{\ell} \\
x\left(T_{\ell-1}\right)=0, \quad x\left(T_{\ell}\right)=0
\end{array}\right.
$$

For our purpose, the upcoming lemma will be a corner stone of the solution of the BVP (7).

Lemma 3.1 Let $\ell \in\{1,2, \ldots, n\}$ be a natural number, $f_{1} \in C\left(J_{\ell} \times \Re \times \Re, \Re\right)$ and there exists a number $\delta \in(0,1)$ such that $t^{\delta} f_{1} \in C\left(J_{\ell} \times \mathfrak{R} \times \mathfrak{R}, \mathfrak{R}\right)$.

Then the function $x \in E_{\ell}$ is a solution of the BVP (7) if and only if $x$ solves the integral equation

$$
x(t)=-\left(T_{\ell}-T_{\ell-1}\right)^{-1}\left(t-T_{\ell-1}\right) I_{T_{\ell-1}^{+}}^{u_{\ell}} y\left(T_{\ell}\right)+I_{T_{\ell-1}^{+}}^{u_{\ell}} y(t),
$$

where

$$
y(t)=f_{1}\left(t,-\left(T_{\ell}-T_{\ell-1}\right)^{-1}\left(t-T_{\ell-1}\right) I_{T_{\ell-1}^{+}}^{u_{\ell}} y\left(T_{\ell}\right)+I_{T_{\ell-1}^{+}}^{u_{\ell}} y(t), y(t)\right), \quad t \in J_{\ell} .
$$


Proof We presume that $x \in E_{\ell}$ is solution of the BVP (7) and we take ${ }^{c} D_{T_{\ell-1}^{+}}^{u_{\ell}} x(t)=y(t)$. Employing the operator $I_{T_{\ell-1}^{+}}^{u_{\ell}}$ to both sides of (7) and regarding Lemma 2.1, we find

$$
x(t)=\omega_{1}+\omega_{2}\left(t-T_{\ell-1}\right)+I_{T_{\ell-1}^{+}}^{u_{\ell}} y(t), \quad t \in J_{\ell} .
$$

By $x\left(T_{\ell-1}\right)=0$, we get $\omega_{1}=0$.

Let $x(t)$ satisfy $x\left(T_{\ell}\right)=0$. So, we observe that

$$
\omega_{2}=-\left(T_{\ell}-T_{\ell-1}\right)^{-1} I_{T_{\ell-1}^{+}}^{u_{\ell}} y\left(T_{\ell}\right)
$$

Then we find

$$
x(t)=-\left(T_{\ell}-T_{\ell-1}\right)^{-1}\left(t-T_{\ell-1}\right) I_{T_{\ell-1}^{+}}^{u_{\ell}} y\left(T_{\ell}\right)+I_{T_{\ell-1}^{+}}^{u_{\ell}} y(t),
$$

where

$$
y(t)=f_{1}\left(t,-\left(T_{\ell}-T_{\ell-1}\right)^{-1}\left(t-T_{\ell-1}\right) I_{T_{\ell-1}^{+}}^{u_{\ell}} y\left(T_{\ell}\right)+I_{T_{\ell-1}^{+}}^{u_{\ell}} y(t), y(t)\right), \quad t \in J_{\ell} .
$$

Conversely, let $x \in E_{\ell}$ be a solution of the integral equation (8). Regarding the continuity of the function $t^{\delta} f_{1}$ and Lemma 2.1, we deduce that $x$ is the solution of the BVP (7).

We will prove the existence result for the BVP (7). This result is based on Theorem 2.1.

Theorem 3.1 Let the conditions of Lemma 3.1 be satisfied and there exist constants $K, L>$ 0 , such that $t^{\delta}\left|f_{1}\left(t, y_{1}, z_{1}\right)-f_{1}\left(t, y_{2}, z_{2}\right)\right| \leq K\left|y_{1}-y_{2}\right|+L\left|z_{1}-z_{2}\right|$, for any $y_{i}, z_{i} \in \Re, i=1,2$, $t \in J_{\ell}$. and the inequality

$$
\frac{2\left(T_{\ell}-T_{\ell-1}\right)^{u_{\ell}-1}\left(T_{\ell}^{1-\delta}-T_{\ell-1}^{1-\delta}\right)}{(1-\delta) \Gamma\left(u_{\ell}\right)}\left(2 K \frac{\left(T_{\ell}-T_{\ell-1}\right)^{u_{\ell}}}{\Gamma\left(u_{\ell}+1\right)}+L\right)<1,
$$

holds.

Then the BVP (7) possesses at least one solution in $E_{\ell}$.

Proof We construct the operators

$$
W_{1}, W_{2}: E_{\ell} \rightarrow E_{\ell}
$$

as follows:

$$
W_{1} y(t)=-\left(T_{\ell}-T_{\ell-1}\right)^{-1}\left(t-T_{\ell-1}\right) I_{T_{\ell-1}^{+}}^{u_{\ell}} y\left(T_{\ell}\right), \quad W_{2} y(t)=I_{T_{\ell-1}^{+}}^{u_{\ell}} y(t),
$$

where

$$
y(t)=f_{1}\left(t,-\left(T_{\ell}-T_{\ell-1}\right)^{-1}\left(t-T_{\ell-1}\right) I_{T_{\ell-1}^{+}}^{u_{\ell}} y\left(T_{\ell}\right)+I_{T_{\ell-1}^{+}}^{u_{\ell}} y(t), y(t)\right), \quad t \in J_{\ell} .
$$

It follows from the properties of fractional integrals and from the continuity of the function $t^{\delta} f_{1}$ that the operators $W_{1}, W_{2}: E_{\ell} \rightarrow E_{\ell}$ defined in (10) are well defined. 
Let

$$
R_{\ell} \geq \frac{\frac{2 f^{\star}\left(T_{\ell}-T_{\ell-1}\right)^{u_{\ell}}}{\Gamma\left(u_{\ell}+1\right)}}{1-\frac{2\left(T_{\ell}-T_{\ell-1}\right)^{u_{\ell}-1}\left(T_{\ell}^{1-\delta}-T_{\ell-1}^{1-\delta}\right)}{(1-\delta) \Gamma\left(u_{\ell}\right)}\left(2 K \frac{\left(T_{\ell}-T_{\ell-1}\right)^{u_{\ell}}}{\Gamma\left(u_{\ell}+1\right)}+L\right)},
$$

where

$$
f^{\star}=\sup _{t \in J_{\ell}}\left|f_{1}(t, 0,0)\right| .
$$

We consider the set

$$
B_{R_{\ell}}=\left\{y \in E_{\ell},\|y\|_{E_{\ell}} \leq R_{\ell}\right\} .
$$

Clearly $B_{R_{\ell}}$ is nonempty, closed, convex and bounded.

Now, we demonstrate that $W_{1}, W_{2}$ satisfy the assumption of Theorem 2.1. We shall prove it in four phases.

STEP 1: Claim: $W_{1}\left(B_{R_{\ell}}\right)+W_{2}\left(B_{R_{\ell}}\right) \subseteq\left(B_{R_{\ell}}\right)$.

For $y \in B_{R_{\ell}}$, we have

$$
\begin{aligned}
\left|\left(W_{1} y\right)(t)+\left(W_{2} y\right)(t)\right| & \\
\leq & \frac{\left(T_{\ell}-T_{\ell-1}\right)^{-1}\left(t-T_{\ell-1}\right)}{\Gamma\left(u_{\ell}\right)} \int_{T_{\ell-1}}^{T_{\ell}}\left(T_{\ell}-s\right)^{u_{\ell}-1} \mid f_{1}\left(s,-\left(T_{\ell}-T_{\ell-1}\right)^{-1}\left(s-T_{\ell-1}\right) I_{T_{\ell-1}^{+}}^{u_{\ell}} y\left(T_{\ell}\right)\right. \\
& \left.+I_{T_{\ell-1}^{+}}^{u_{\ell}} y(s), y(s)\right) \mid d s \\
& +\frac{1}{\Gamma\left(u_{\ell}\right)} \int_{T_{\ell-1}}^{t}(t-s)^{u_{\ell}-1} \mid f_{1}\left(s,-\left(T_{\ell}-T_{\ell-1}\right)^{-1}\left(s-T_{\ell-1}\right) I_{T_{\ell-1}^{+}}^{u_{\ell}} y\left(T_{\ell}\right)\right. \\
& \left.+I_{T_{\ell-1}^{+}}^{u_{\ell}} y(s), y(s)\right) \mid d s \\
\leq & \frac{2}{\Gamma\left(u_{\ell}\right)} \int_{T_{\ell-1}}^{T_{\ell}}\left(T_{\ell}-s\right)^{u_{\ell}-1} \mid f_{1}\left(s,-\left(T_{\ell}-T_{\ell-1}\right)^{-1}\left(s-T_{\ell-1}\right) I_{T_{\ell-1}^{+}}^{u_{\ell}} y\left(T_{\ell}\right)\right. \\
& \left.+I_{T_{\ell-1}^{+}}^{u_{\ell}} y(s), y(s)\right) \mid d s \\
\leq & \frac{2}{\Gamma\left(u_{\ell}\right)} \int_{T_{\ell-1}}^{T_{\ell}}\left(T_{\ell}-s\right)^{u_{\ell}-1} \mid f_{1}\left(s,-\left(T_{\ell}-T_{\ell-1}\right)^{-1}\left(s-T_{\ell-1}\right) I_{T_{\ell-1}^{+}}^{u_{\ell}} y\left(T_{\ell}\right)\right. \\
& \left.+I_{T_{\ell-1}^{+}}^{u_{\ell}} y(s), y(s)\right)-f_{1}(s, 0,0) \mid d s \\
& +\frac{2}{\Gamma\left(u_{\ell}\right)} \int_{T_{\ell-1}}^{T_{\ell}}\left(T_{\ell}-s\right)^{u_{\ell}-1}\left|f_{1}(s, 0,0)\right| d s \\
\leq & \frac{2}{\Gamma\left(u_{\ell}\right)} \int_{T_{\ell-1}}^{T_{\ell}}\left(T_{\ell}-s\right)^{u_{\ell}-1} s^{-\delta}\left(K\left|-\left(T_{\ell}-T_{\ell-1}\right)^{-1}\left(s-T_{\ell-1}\right) I_{T_{\ell-1}^{+}}^{u_{\ell}} y\left(T_{\ell}\right)+I_{T_{\ell-1}^{+}}^{u_{\ell}} y(s)\right|\right. \\
& +L|y(s)|) d s+\frac{2 f^{\star}\left(T_{\ell}-T_{\ell-1}\right)^{u_{\ell}}}{\Gamma\left(u_{\ell}+1\right)} \\
\leq & \frac{2\left(T_{\ell}-T_{\ell-1}\right)^{u_{\ell}-1}}{\Gamma\left(u_{\ell}\right)} \int_{T_{\ell-1}}^{T_{\ell}} s^{-\delta}\left(K\left|I_{T_{\ell-1}^{+}}^{u_{\ell}} y\left(T_{\ell}\right)+I_{T_{\ell-1}^{+}}^{u_{\ell}} y(s)\right|+L|y(s)|\right) d s \\
& +\frac{2 f^{\star}\left(T_{\ell}-T_{\ell-1}\right)^{u_{\ell}}}{\Gamma\left(u_{\ell}+1\right)}
\end{aligned}
$$




$$
\begin{aligned}
& \leq \frac{2\left(T_{\ell}-T_{\ell-1}\right)^{u_{\ell}-1}}{\Gamma\left(u_{\ell}\right)}\left(2 K\left\|I_{T_{\ell-1}^{+}}^{u_{\ell}} y\right\|_{E_{\ell}}+L\|y\|_{E_{\ell}}\right) \int_{T_{\ell-1}}^{T_{\ell}} s^{-\delta} d s+\frac{2 f^{\star}\left(T_{\ell}-T_{\ell-1}\right)^{u_{\ell}}}{\Gamma\left(u_{\ell}+1\right)} \\
& \leq \frac{2\left(T_{\ell}-T_{\ell-1}\right)^{u_{\ell}-1}\left(T_{\ell}^{1-\delta}-T_{\ell-1}^{1-\delta}\right)}{(1-\delta) \Gamma\left(u_{\ell}\right)}\left(2 K \frac{\left(T_{\ell}-T_{\ell-1}\right)^{u_{\ell}}}{\Gamma\left(u_{\ell}+1\right)}+L\right) R_{\ell}+\frac{2 f^{\star}\left(T_{\ell}-T_{\ell-1}\right)^{u_{\ell}}}{\Gamma\left(u_{\ell}+1\right)} \\
& \leq R_{\ell},
\end{aligned}
$$

which means that $W_{1}\left(B_{R_{\ell}}\right)+W_{2}\left(B_{R_{\ell}}\right) \subseteq B_{R_{\ell}}$.

STEP 2: Claim: $W_{1}$ is continuous.

We presume that the sequence $\left(y_{n}\right)$ converges to $y$ in $E_{\ell}$ and $t \in J_{\ell}$. Then

$$
\begin{aligned}
&\left|\left(W_{1} y_{n}\right)(t)-\left(W_{1} y\right)(t)\right| \\
& \leq \frac{\left(T_{\ell}-T_{\ell-1}\right)^{-1}\left(t-T_{\ell-1}\right)}{\Gamma\left(u_{\ell}\right)} \\
& \quad \times \int_{T_{\ell-1}}^{T_{\ell}}\left(T_{\ell}-s\right)^{u_{\ell}-1} \mid f_{1}\left(s,-\left(T_{\ell}-T_{\ell-1}\right)^{-1}\left(s-T_{\ell-1}\right) I_{T_{\ell-1}^{+}}^{u_{\ell}} y_{n}\left(T_{\ell}\right)+I_{T_{\ell-1}^{+}}^{u_{\ell}} y_{n}(s), y_{n}(s)\right) \\
& \quad-f_{1}\left(s,-\left(T_{\ell}-T_{\ell-1}\right)^{-1}\left(s-T_{\ell-1}\right) I_{T_{\ell-1}^{+}}^{u_{\ell}} y\left(T_{\ell}\right)+I_{T_{\ell-1}^{+}}^{u_{\ell}} y(s), y(s)\right) \mid d s \\
& \leq \\
& \quad \frac{\left(T_{\ell}-T_{\ell-1}\right)^{-1}\left(t-T_{\ell-1}\right)}{\Gamma\left(u_{\ell}\right)} \\
& \quad \times \int_{T_{\ell-1}}^{T_{\ell}}\left(T_{\ell}-s\right)^{u_{\ell}-1} s^{-\delta}\left(K \mid-\left(T_{\ell}-T_{\ell-1}\right)^{-1}\left(s-T_{\ell-1}\right) I_{T_{\ell-1}^{+}}^{u_{\ell}}\left(y_{n}\left(T_{\ell}\right)-y\left(T_{\ell}\right)\right)\right. \\
&\left.\quad+I_{T_{\ell-1}^{+}}^{u_{\ell}}\left(y_{n}(s)-y(s)\right)|+L|\left(y_{n}(s)-y(s)\right) \mid\right) \\
& \leq \frac{\left(T_{\ell}-T_{\ell-1}\right)^{u_{\ell}-1}}{\Gamma\left(u_{\ell}\right)} \int_{T_{\ell-1}}^{T_{\ell}} s^{-\delta}\left(K \mid I_{T_{\ell-1}^{+}}^{u_{\ell}}\left(y_{n}\left(T_{\ell}\right)-y\left(T_{\ell}\right)\right)\right. \\
&\left.+I_{T_{\ell-1}^{+}}^{u_{\ell}}\left(y_{n}(s)-y(s)\right)|+L|\left(y_{n}(s)-y(s)\right) \mid\right) \\
& \leq \frac{\left(T_{\ell}-T_{\ell-1}\right)^{u_{\ell}-1}}{\Gamma\left(u_{\ell}\right)}\left(2 K\left\|I_{T_{\ell-1}^{+}}^{u_{\ell}}\left(y_{n}-y\right)\right\|_{E_{\ell}}+L\left\|y_{n}-y\right\|_{E_{\ell}}\right) \int_{T_{\ell-1}}^{T_{\ell}} s^{-\delta} d s \\
& \leq \frac{\left(T_{\ell}-T_{\ell-1}\right)^{u_{\ell}-1}\left(T_{\ell}^{1-\delta}-T_{\ell-1}^{1-\delta}\right)}{(1-\delta) \Gamma\left(u_{\ell}\right)}\left(2 K \frac{\left(T_{\ell}-T_{\ell-1}\right)^{u_{\ell}}}{\Gamma\left(u_{\ell}+1\right)}+L\right)\left\|y_{n}-y\right\|_{E_{\ell}}
\end{aligned}
$$

i.e., we obtain

$$
\left\|\left(W_{1} y_{n}\right)-\left(W_{1} y\right)\right\|_{E_{\ell}} \rightarrow 0 \quad \text { as } n \rightarrow \infty
$$

Ergo, the operator $W_{1}$ is a continuous on $E_{\ell}$.

STEP 3: $W_{1}$ is compact

Now, we will show that $W_{1}\left(B_{R_{\ell}}\right)$ is relatively compact, meaning that $W_{1}$ is compact. Clearly $W_{1}\left(B_{R_{\ell}}\right)$ is uniformly bounded because by Step 1 , we have $W_{1}\left(B_{R_{\ell}}\right)=\left\{W_{1}(y): y \in\right.$ $\left.B_{R_{\ell}}\right\} \subset W_{1}\left(B_{R_{\ell}}\right)+W_{2}\left(B_{R_{\ell}}\right) \subseteq\left(B_{R_{\ell}}\right)$ thus for each $y \in B_{R_{\ell}}$ we have $\left\|W_{1}(y)\right\|_{E_{\ell}} \leq R_{\ell}$, which means that $W_{1}\left(B_{R_{\ell}}\right)$ is bounded. It remains to show that $W_{1}\left(B_{R_{\ell}}\right)$ is equicontinuous.

For $t_{1}, t_{2} \in J_{\ell}, t_{1}<t_{2}$ and $y \in B_{R_{\ell}}$, we have

$$
\left|\left(W_{1} y\right)\left(t_{2}\right)-\left(W_{1} y\right)\left(t_{1}\right)\right|
$$




$$
\begin{aligned}
& =\mid-\frac{\left(T_{\ell}-T_{\ell-1}\right)^{-1}\left(t_{2}-T_{\ell-1}\right)}{\Gamma\left(u_{\ell}\right)} \int_{T_{\ell-1}}^{T_{\ell}}\left(T_{\ell}-s\right)^{u_{\ell}-1} f_{1}\left(s,-\left(T_{\ell}-T_{\ell-1}\right)^{-1}\left(s-T_{\ell-1}\right) I_{T_{\ell-1}^{+}}^{u_{\ell}} y\left(T_{\ell}\right)\right. \\
& \left.+I_{T_{\ell-1}^{+}}^{u_{\ell}} y(s), y(s)\right) d s+\frac{\left(T_{\ell}-T_{\ell-1}\right)^{-1}\left(t_{1}-T_{\ell-1}\right)}{\Gamma\left(u_{\ell}\right)} \\
& \times \int_{T_{\ell-1}}^{T_{\ell}}\left(T_{\ell}-s\right)^{u_{\ell}-1} f_{1}\left(s,-\left(T_{\ell}-T_{\ell-1}\right)^{-1}\left(s-T_{\ell-1}\right) I_{T_{\ell-1}^{+}}^{u_{\ell}} y\left(T_{\ell}\right)+I_{T_{\ell-1}^{+}}^{u_{\ell}} y(s), y(s)\right) d s \mid \\
& \leq \frac{\left(T_{\ell}-T_{\ell-1}\right)^{-1}}{\Gamma\left(u_{\ell}\right)}\left(\left(t_{2}-T_{\ell-1}\right)-\left(t_{1}-T_{\ell-1}\right)\right) \\
& \times \int_{T_{\ell-1}}^{T_{\ell}}\left(T_{\ell}-s\right)^{u_{\ell}-1}\left|f_{1}\left(s,-\left(T_{\ell}-T_{\ell-1}\right)^{-1}\left(s-T_{\ell-1}\right) I_{T_{\ell-1}^{+}}^{u_{\ell}} y\left(T_{\ell}\right)+I_{T_{\ell-1}^{+}}^{u_{\ell}} y(s), y(s)\right)\right| d s \\
& \leq \frac{\left(T_{\ell}-T_{\ell-1}\right)^{u_{\ell}-2}}{\Gamma\left(u_{\ell}\right)}\left(\left(t_{2}-T_{\ell-1}\right)-\left(t_{1}-T_{\ell-1}\right)\right) \\
& \times \int_{T_{\ell-1}}^{T_{\ell}}\left|f_{1}\left(s,-\left(T_{\ell}-T_{\ell-1}\right)^{-1}\left(s-T_{\ell-1}\right) I_{T_{\ell-1}^{+}}^{u_{\ell}} y\left(T_{\ell}\right)+I_{T_{\ell-1}^{+}}^{u_{\ell}} y(s), y(s)\right)-f_{1}(s, 0,0)\right| d s \\
& +\frac{\left(T_{\ell}-T_{\ell-1}\right)^{u_{\ell}-2}}{\Gamma\left(u_{\ell}\right)}\left(\left(t_{2}-T_{\ell-1}\right)-\left(t_{1}-T_{\ell-1}\right)\right) \int_{T_{\ell-1}}^{T_{\ell}}\left|f_{1}(s, 0,0)\right| d s \\
& \leq \frac{\left(T_{\ell}-T_{\ell-1}\right)^{u_{\ell}-2}}{\Gamma\left(u_{\ell}\right)}\left(\left(t_{2}-T_{\ell-1}\right)-\left(t_{1}-T_{\ell-1}\right)\right) \\
& \left.\times \int_{T_{\ell-1}}^{T_{\ell}} s^{-\delta}\left(K\left|-\left(T_{\ell}-T_{\ell-1}\right)^{-1}\left(s-T_{\ell-1}\right) I_{T_{\ell-1}^{+}}^{u_{\ell}} y\left(T_{\ell}\right)+I_{T_{\ell-1}^{+}}^{u_{\ell}} y(s)\right|+L|y(s)|\right)\right) d s \\
& +\frac{f^{\star}\left(T_{\ell}-T_{\ell-1}\right)^{u_{\ell}-1}}{\Gamma\left(u_{\ell}\right)}\left(\left(t_{2}-T_{\ell-1}\right)-\left(t_{1}-T_{\ell-1}\right)\right) \\
& \leq \frac{\left(T_{\ell}-T_{\ell-1}\right)^{u_{\ell}-2}}{\Gamma\left(u_{\ell}\right)}\left(\left(t_{2}-T_{\ell-1}\right)-\left(t_{1}-T_{\ell-1}\right)\right) \\
& \left.\times \int_{T_{\ell-1}}^{T_{\ell}} s^{-\delta}\left(K\left|I_{T_{\ell-1}^{+}}^{u_{\ell}} y\left(T_{\ell}\right)+I_{T_{\ell-1}^{+}}^{u_{\ell}} y(s)\right|+L|y(s)|\right)\right) d s \\
& +\frac{f^{\star}\left(T_{\ell}-T_{\ell-1}\right)^{u_{\ell}-1}}{\Gamma\left(u_{\ell}\right)}\left(\left(t_{2}-T_{\ell-1}\right)-\left(t_{1}-T_{\ell-1}\right)\right) \\
& \leq \frac{\left(T_{\ell}-T_{\ell-1}\right)^{u_{\ell}-2}}{\Gamma\left(u_{\ell}\right)}\left(\left(t_{2}-T_{\ell-1}\right)-\left(t_{1}-T_{\ell-1}\right)\right)\left(2 K\left\|I_{T_{\ell-1}^{+}}^{u_{\ell}} y\right\|_{E_{\ell}}+L\|y\|_{E_{\ell}}\right) \int_{T_{\ell-1}}^{T_{\ell}} s^{-\delta} d s \\
& +\frac{f^{\star}\left(T_{\ell}-T_{\ell-1}\right)^{u_{\ell}-1}}{\Gamma\left(u_{\ell}\right)}\left(\left(t_{2}-T_{\ell-1}\right)-\left(t_{1}-T_{\ell-1}\right)\right) \\
& \leq \frac{\left(T_{\ell}-T_{\ell-1}\right)^{u_{\ell}-2}\left(T_{\ell}^{1-\delta}-T_{\ell-1}^{1-\delta}\right)}{(1-\delta) \Gamma\left(u_{\ell}\right)}\left(\left(t_{2}-T_{\ell-1}\right)-\left(t_{1}-T_{\ell-1}\right)\right) \\
& \times\left(2 K \frac{\left(T_{\ell}-T_{\ell-1}\right)^{u_{\ell}}}{\Gamma\left(u_{\ell}+1\right)}+L\right)\|y\|_{E_{\ell}} \\
& +\frac{f^{\star}\left(T_{\ell}-T_{\ell-1}\right)^{u_{\ell}-1}}{\Gamma\left(u_{\ell}\right)}\left(\left(t_{2}-T_{\ell-1}\right)-\left(t_{1}-T_{\ell-1}\right)\right) \\
& \leq\left[\frac{\left(T_{\ell}-T_{\ell-1}\right)^{u_{\ell}-2}\left(T_{\ell}^{1-\delta}-T_{\ell-1}^{1-\delta}\right)}{(1-\delta) \Gamma\left(u_{\ell}\right)}\left(2 K \frac{\left(T_{\ell}-T_{\ell-1}\right)^{u_{\ell}}}{\Gamma\left(u_{\ell}+1\right)}+L\right)\|y\|_{E_{\ell}}\right. \\
& \left.+\frac{f^{\star}\left(T_{\ell}-T_{\ell-1}\right)^{u_{\ell}-1}}{\Gamma\left(u_{\ell}\right)}\right]
\end{aligned}
$$




$$
\times\left(\left(t_{2}-T_{\ell-1}\right)-\left(t_{1}-T_{\ell-1}\right)\right)
$$

Hence $\left\|\left(W_{1} y\right)\left(t_{2}\right)-\left(W_{1} y\right)\left(t_{1}\right)\right\|_{E_{\ell}} \rightarrow 0$ as $\left|t_{2}-t_{1}\right| \rightarrow 0$. It implies that $W_{1}\left(B_{R_{\ell}}\right)$ is equicontinuous.

STEP 4: $W_{2}$ is a strict contraction

For $x(t), y(t) \in E_{\ell}$, we obtain

$$
\begin{aligned}
\left|\left(W_{2} x\right)(t)-\left(W_{2} y\right)(t)\right| & \mid \frac{1}{\Gamma\left(u_{\ell}\right)} \int_{T_{\ell-1}}^{t}(t-s)^{u_{\ell}-1} f_{1}\left(s,-\left(T_{\ell}-T_{\ell-1}\right)^{-1}\left(s-T_{\ell-1}\right) I_{T_{\ell-1}^{+}}^{u_{\ell}} x\left(T_{\ell}\right)+I_{T_{\ell-1}^{+}}^{u_{\ell}} x(s), x(s)\right) d s \\
& -\frac{1}{\Gamma\left(u_{\ell}\right)} \int_{T_{\ell-1}}^{t}(t-s)^{u_{\ell}-1} f_{1}\left(s,-\left(T_{\ell}-T_{\ell-1}\right)^{-1}\left(s-T_{\ell-1}\right) I_{T_{\ell-1}^{+}}^{u_{\ell}} y\left(T_{\ell}\right)+I_{T_{\ell-1}^{+}}^{u_{\ell}} y(s), y(s)\right) d s \mid \\
\leq & \frac{1}{\Gamma\left(u_{\ell}\right)} \int_{T_{\ell-1}}^{t}(t-s)^{u_{\ell}-1} \mid f_{1}\left(s,-\left(T_{\ell}-T_{\ell-1}\right)^{-1}\left(s-T_{\ell-1}\right) I_{T_{\ell-1}^{+}}^{u_{\ell}} x\left(T_{\ell}\right)+I_{T_{\ell-1}^{+}}^{u_{\ell}} x(s), x(s)\right) \\
& -f_{1}\left(s,-\left(T_{\ell}-T_{\ell-1}\right)^{-1}\left(s-T_{\ell-1}\right) I_{T_{\ell-1}^{+}}^{u_{\ell}} y\left(T_{\ell}\right)+I_{T_{\ell-1}^{+}}^{u_{\ell}} y(s), y(s)\right) \mid d s \\
\leq & \frac{\left(T_{\ell}-T_{\ell-1}\right)^{u_{\ell}-1}}{\Gamma\left(u_{\ell}\right)} \int_{T_{\ell-1}}^{t} s^{-\delta}\left(K \mid\left(T_{\ell}-T_{\ell-1}\right)^{-1}\left(s-T_{\ell-1}\right)\left(I_{T_{\ell-1}^{+}}^{u_{\ell}}(x-y)\left(T_{\ell}\right)\right)\right. \\
& \left.+\left(I_{T_{\ell-1}^{+}}^{u_{\ell}}(x-y)(s)\right)|+L|(x-y)(s) \mid\right) d s \\
\leq & \frac{\left(T_{\ell}-T_{\ell-1}\right)^{u_{\ell}-1}}{\Gamma\left(u_{\ell}\right)} \int_{T_{\ell-1}}^{t} s^{-\delta}\left(K \mid\left(I_{T_{\ell-1}^{+}}^{u_{\ell}}(x-y)\left(T_{\ell}\right)+I_{T_{\ell-1}^{+}}^{u_{\ell}}(x-y)(s)|+L|(x-y)(s) \mid\right) d s\right. \\
\leq & \frac{\left(T_{\ell}-T_{\ell-1}\right)^{u_{\ell}-1}}{\Gamma\left(u_{\ell}\right)}\left(2 K \|\left(I_{T_{\ell-1}^{+}}^{u_{\ell}}(x-y)\left\|_{E_{\ell}}+L\right\| x-y \|_{E_{\ell}}\right) \int_{T_{\ell-1}}^{t} s^{-\delta} d s\right. \\
\leq & \frac{\left(T_{\ell}-T_{\ell-1}\right)^{u_{\ell}-1}\left(T_{\ell}^{1-\delta}-T_{\ell-1}^{1-\delta}\right)}{(1-\delta) \Gamma\left(u_{\ell}\right)}\left(2 K \frac{\left(T_{\ell}-T_{\ell-1}\right)_{\ell \ell}^{u_{\ell}}}{\Gamma\left(u_{\ell}+1\right)}+L\right)\|x-y\|_{E_{\ell}} .
\end{aligned}
$$

Consequently by (9), the operator $W_{2}$ is a strict contraction.

Therefore, all conditions of Theorem 2.1 are fulfilled and thus there exists $\widetilde{x_{\ell}} \in B_{R_{\ell}}$, such that $W_{1} \tilde{x_{\ell}}+W_{2} \tilde{x_{\ell}}=\tilde{x_{\ell}}$, which is a solution of the BVP (7). Since $B_{R_{\ell}} \subset E_{\ell}$, the claim of Theorem 3.1 is proved.

Now, we will prove the existence result for the BVP (1).

Introduce the following assumption:

(H2) Let $f_{1} \in C(J \times \Re \times \Re, \Re)$ and there exists a number $\delta \in(0,1)$ such that $t^{\delta} f_{1} \in C(J \times$ $\Re \times \Re, \Re)$ and there exist constants $K, L>0$, such that $t^{\delta}\left|f_{1}\left(t, y_{1}, z_{1}\right)-f_{1}\left(t, y_{2}, z_{2}\right)\right| \leq$ $K\left|y_{1}-y_{2}\right|+L\left|z_{1}-z_{2}\right|$, for any $y_{1}, y_{2}, z_{1}, z_{2} \in \Re$ and $t \in J$.

Theorem 3.2 Let the conditions (H1), (H2) and inequality (9) be satisfied for all $\ell \in$ $\{1,2, \ldots, n\}$.

Then the problem (1) possesses at least one solution in $C(J, \Re)$.

Proof For any $\ell \in\{1,2, \ldots, n\}$ according to Theorem 3.1 the BVP (7) possesses at least one solution $\tilde{x_{\ell}} \in E_{\ell}$. 
For any $\ell \in\{1,2, \ldots, n\}$ we define the function

$$
x_{\ell}= \begin{cases}0, & t \in\left[0, T_{\ell-1}\right] \\ \tilde{x}_{\ell}, & t \in J_{\ell}\end{cases}
$$

Thus, the function $x_{\ell} \in C\left(\left[0, T_{\ell}\right], \Re\right)$ solves the integral equation (6) for $t \in J_{\ell}$ with $x_{\ell}(0)=$ $0, x_{\ell}\left(T_{\ell}\right)=\tilde{x}_{\ell}\left(T_{\ell}\right)=0$.

Then the function

$$
x(t)=\left\{\begin{array}{l}
x_{1}(t), \\
x_{2}(t)= \begin{cases}0, & t \in J_{1}, \\
\tilde{x}_{2}, & t \in J_{2},\end{cases} \\
\vdots \\
x_{n}(t)= \begin{cases}0, & t \in\left[0, T_{1-1}\right], \\
\tilde{x}_{\ell}, & t \in J_{\ell},\end{cases}
\end{array}\right.
$$

is a solution of the $\operatorname{BVP}(1)$ in $C(J, \Re)$.

\section{Ulam-Hyers stability}

Theorem 4.1 Let the conditions (H1), (H2) and inequality (9) be satisfied. Then BVP (1) is $(\mathrm{UH})$ stable.

Proof Let $\epsilon>0$ an arbitrary number and the function $z(t)$ from $z \in C\left(J_{\ell}, \Re\right)$ satisfy inequality (4).

For any $\ell \in\{1,2, \ldots, n\}$ we define the functions $z_{1}(t) \equiv z(t), t \in\left[0, T_{1}\right]$ and for $\ell=$ $2,3, \ldots, n$ :

$$
z_{\ell}(t)= \begin{cases}0, & t \in\left[0, T_{\ell-1}\right] \\ z(t), & t \in J_{\ell}\end{cases}
$$

For any $\ell \in\{1,2, \ldots, n\}$ according to equality (5) for $t \in J$ we get

$$
{ }^{c} D_{T_{\ell-1}+}^{u(t)} z_{\ell}(t)=\int_{T_{\ell-1}}^{t} \frac{(t-s)^{1-u_{\ell}}}{\Gamma\left(2-u_{\ell}\right)} z^{(2)}(s) d s .
$$

Taking the (CFI) $I_{T_{\ell-1}^{+}}^{u_{\ell}}$ of both sides of the inequality (4), we obtain

$$
\begin{aligned}
\mid z_{\ell}(t) & +\frac{\left(T_{\ell}-T_{\ell-1}\right)^{-1}\left(t-T_{\ell-1}\right)}{\Gamma\left(u_{\ell}\right)} \\
& \times \int_{T_{\ell-1}}^{T_{\ell}}\left(T_{\ell}-s\right)^{u_{\ell-1}} f_{1}\left(s,-\left(T_{\ell}-T_{\ell-1}\right)^{-1}\left(s-T_{\ell-1}\right) I_{T_{\ell-1}^{+}}^{u_{\ell}} z_{\ell}\left(T_{\ell}\right)+I_{T_{\ell-1}^{+}}^{u_{\ell}} z_{\ell}(s), z_{\ell}(s)\right) d s \\
& -\frac{1}{\Gamma\left(u_{\ell}\right)} \int_{T_{\ell-1}}^{t}(t-s)^{u_{\ell-1}} f_{1}\left(s,-\left(T_{\ell}-T_{\ell-1}\right)^{-1}\left(s-T_{\ell-1}\right) I_{T_{\ell-1}^{+}}^{u_{\ell}} z_{\ell}\left(T_{\ell}\right)\right. \\
& \left.+I_{T_{\ell-1}^{+}}^{u_{\ell}} z_{\ell}(s), z_{\ell}(s)\right) d s \mid
\end{aligned}
$$




$$
\begin{aligned}
& \leq \epsilon \int_{T_{\ell-1}}^{t} \frac{(t-s)^{u_{\ell}-1}}{\Gamma\left(u_{\ell}\right)} d s \\
& \leq \epsilon \frac{\left(T_{\ell}-T_{\ell-1}\right)^{u_{\ell}}}{\Gamma\left(u_{\ell}+1\right)} .
\end{aligned}
$$

According to Theorem 3.2, BVP (1) has a solution $x \in C(J, \Re)$ defined by $x(t)=x_{\ell}(t)$ for $t \in J_{\ell}, \ell=1,2, \ldots, n$, where

$$
x_{\ell}= \begin{cases}0, & t \in\left[0, T_{\ell-1}\right], \\ \tilde{x}_{\ell}, & t \in J_{\ell},\end{cases}
$$

and $\widetilde{x}_{\ell} \in E_{\ell}$ is a solution of (7). According to Lemma 3.1 the integral equation

$$
\begin{aligned}
\tilde{x}_{\ell}(t)= & -\frac{\left(T_{\ell}-T_{\ell-1}\right)^{-1}\left(t-T_{\ell-1}\right)}{\Gamma\left(u_{\ell}\right)} \\
& \times \int_{T_{\ell-1}}^{T_{\ell}}\left(T_{\ell}-s\right)^{u_{\ell-1}} f_{1}\left(s,-\left(T_{\ell}-T_{\ell-1}\right)^{-1}\left(s-T_{\ell-1}\right) I_{T_{\ell-1}^{+}}^{u_{\ell}} \tilde{x}_{\ell}\left(T_{\ell}\right)+I_{T_{\ell-1}^{+}}^{u_{\ell}} \tilde{x}_{\ell}(s), \tilde{x}_{\ell}(s)\right) d s \\
& +\frac{1}{\Gamma\left(u_{\ell}\right)} \int_{T_{\ell-1}}^{t}(t-s)^{u_{\ell-1}} f_{1}\left(s,-\left(T_{\ell}-T_{\ell-1}\right)^{-1}\left(s-T_{\ell-1}\right) I_{T_{\ell-1}^{+}}^{u_{\ell}} \tilde{x}_{\ell}\left(T_{\ell}\right)\right. \\
& \left.+I_{T_{\ell-1}^{+}}^{u_{\ell}} \tilde{x}_{\ell}(s), \tilde{x}_{\ell}(s)\right) d s
\end{aligned}
$$

holds.

Let $t \in J_{\ell}, \ell=1,2, \ldots, n$. Then by Eqs. (12) and (13) we get

$$
\begin{aligned}
\mid z(t) & -x(t) \mid \\
= & \left|z(t)-x_{\ell}(t)\right| \\
= & \left|z_{\ell}(t)-\tilde{x}_{\ell}(t)\right| \\
= & \mid z_{\ell}(t)+\frac{\left(T_{\ell}-T_{\ell-1}\right)^{-1}\left(t-T_{\ell-1}\right)}{\Gamma\left(u_{\ell}\right)} \\
& \times \int_{T_{\ell-1}}^{T_{\ell}}\left(T_{\ell}-s\right)^{u_{\ell-1}} f_{1}\left(s,-\left(T_{\ell}-T_{\ell-1}\right)^{-1}\left(s-T_{\ell-1}\right) I_{T_{\ell-1}^{+}}^{u_{\ell}} \tilde{x}_{\ell}\left(T_{\ell}\right)\right. \\
& \left.+I_{T_{\ell-1}^{+}}^{u_{\ell}} \tilde{x}_{\ell}(s), \tilde{x}_{\ell}(s)\right) d s \\
& -\frac{1}{\Gamma\left(u_{\ell}\right)} \int_{T_{\ell-1}}^{t}(t-s)^{u_{\ell-1}} f_{1}\left(s,-\left(T_{\ell}-T_{\ell-1}\right)^{-1}\left(s-T_{\ell-1}\right) I_{T_{\ell-1}^{+}}^{u_{\ell}} \tilde{x}_{\ell}\left(T_{\ell}\right)\right. \\
& \left.+I_{T_{\ell-1}^{+}}^{u_{\ell}} \tilde{x}_{\ell}(s), \tilde{x}_{\ell}(s)\right) d s \mid \\
& +\frac{\left(T_{\ell}-T_{\ell-1}\right)^{-1}\left(t-T_{\ell-1}\right)}{\Gamma\left(u_{\ell}\right)} \\
& \times \int_{T_{\ell-1}}^{T_{\ell}}\left(T_{\ell}-s\right)^{u_{\ell}-1} \mid f_{1}\left(s,-\left(T_{\ell}-T_{\ell-1}\right)^{-1}\left(s-T_{\ell-1}\right) I_{T_{\ell-1}^{+}}^{u_{\ell}} z_{\ell}\left(T_{\ell}\right)\right. \\
& \left.+I_{T_{\ell-1}^{+}}^{u_{\ell}} z_{\ell}(s), z_{\ell}(s)\right) d s-f_{1}\left(s,-\left(T_{\ell}-T_{\ell-1}\right)^{-1}\left(s-T_{\ell-1}\right) I_{T_{\ell-1}^{+}}^{u_{\ell}} \tilde{x}_{\ell}\left(T_{\ell}\right)\right. \\
& \left.+I_{T_{\ell-1}^{+}}^{u_{\ell}} \tilde{x}_{\ell}(s), \tilde{x}_{\ell}(s)\right) \mid d s \\
&
\end{aligned}
$$




$$
\begin{aligned}
& +\frac{1}{\Gamma\left(u_{\ell}\right)} \int_{T_{\ell-1}}^{t}(t-s)^{u_{\ell}-1} \mid f_{1}\left(s,-\left(T_{\ell}-T_{\ell-1}\right)^{-1}\left(s-T_{\ell-1}\right) I_{T_{\ell-1}^{+}}^{u_{\ell}} z_{\ell}\left(T_{\ell}\right)\right. \\
& \left.+I_{T_{\ell-1}^{+}}^{u_{\ell}} z_{\ell}(s), z_{\ell}(s)\right) d s \\
& -f_{1}\left(s,-\left(T_{\ell}-T_{\ell-1}\right)^{-1}\left(s-T_{\ell-1}\right) I_{T_{\ell-1}^{+}}^{u_{\ell}} \tilde{x}_{\ell}\left(T_{\ell}\right)+I_{T_{\ell-1}^{+}}^{u_{\ell}} \tilde{x}_{\ell}(s), \tilde{x}_{\ell}(s)\right) \mid d s \\
& \leq \epsilon \frac{\left(T_{\ell}-T_{\ell-1}\right)^{u_{\ell}}}{\Gamma\left(u_{\ell}+1\right)}+\frac{\left(T_{\ell}-T_{\ell-1}\right)^{-1}\left(t-T_{\ell-1}\right)}{\Gamma\left(u_{\ell}\right)} \\
& \times \int_{T_{\ell-1}}^{T_{\ell}}\left(T_{\ell}-s\right)^{u_{\ell}-1} s^{-\delta}\left(K \mid\left(T_{\ell}-T_{\ell-1}\right)^{-1}\left(s-T_{\ell-1}\right)\left(I_{T_{\ell-1}^{+}}^{u_{\ell}}\left(z_{\ell}\left(T_{\ell}\right)-\tilde{x}_{\ell}\left(T_{\ell}\right)\right)\right)\right. \\
& \left.+\left(I_{T_{\ell-1}^{+}}^{u_{\ell}}\left(z_{\ell}(s)-\widetilde{x}_{\ell}(s)\right)\right)|+L|\left(z_{\ell}(s)-\widetilde{x}_{\ell}(s)\right) \mid\right) d s \\
& +\frac{1}{\Gamma\left(u_{\ell}\right)} \int_{T_{\ell-1}}^{t}(t-s)^{u_{\ell}-1} s^{-\delta}\left(K \mid\left(T_{\ell}-T_{\ell-1}\right)^{-1}\left(s-T_{\ell-1}\right)\left(I_{T_{\ell-1}^{+}}^{u_{\ell}}\left(z_{\ell}\left(T_{\ell}\right)-\widetilde{x}_{\ell}\left(T_{\ell}\right)\right)\right)\right. \\
& \left.+\left(I_{T_{\ell-1}^{+}}^{u_{\ell}}\left(z_{\ell}(s)-\tilde{x}_{\ell}(s)\right)\right)|+L|\left(z_{\ell}(s)-\tilde{x}_{\ell}(s)\right) \mid\right) d s \\
& \leq \epsilon \frac{\left(T_{\ell}-T_{\ell-1}\right)^{u_{\ell}}}{\Gamma\left(u_{\ell}+1\right)}+\frac{2\left(T_{\ell}-T_{\ell-1}\right)^{u_{\ell}-1}}{\Gamma\left(u_{\ell}\right)} \\
& \times \int_{T_{\ell-1}}^{T_{\ell}} s^{-\delta}\left(K\left|\left(I_{T_{\ell-1}^{+}}^{u_{\ell}}\left(z_{\ell}\left(T_{\ell}\right)-\tilde{x}_{\ell}\left(T_{\ell}\right)\right)\right)+\left(I_{T_{\ell-1}^{+}}^{u_{\ell}}\left(z_{\ell}(s)-\tilde{x}_{\ell}(s)\right)\right)\right|\right. \\
& \left.+L\left|\left(z_{\ell}(s)-\tilde{x}_{\ell}(s)\right)\right|\right) d s \\
& \leq \epsilon \frac{\left(T_{\ell}-T_{\ell-1}\right)^{u_{\ell}}}{\Gamma\left(u_{\ell}+1\right)} \\
& +\frac{2\left(T_{\ell}-T_{\ell-1}\right)^{u_{\ell}-1}}{\Gamma\left(u_{\ell}\right)}\left(2 K\left\|I_{T_{\ell-1}^{+}}^{u_{\ell}}\left(z_{\ell}-\tilde{x}_{\ell}\right)\right\|_{E_{\ell}}+L\left\|z_{\ell}-\tilde{x}_{\ell}\right\|_{E_{\ell}}\right) \int_{T_{\ell-1}}^{T_{\ell}} s^{-\delta} d s \\
& \leq \epsilon \frac{\left(T_{\ell}-T_{\ell-1}\right)^{u_{\ell}}}{\Gamma\left(u_{\ell}+1\right)}+\frac{2\left(T_{\ell}-T_{\ell-1}\right)^{u_{\ell}-1}\left(T_{\ell}^{1-\delta}-T_{\ell-1}^{1-\delta}\right)}{(1-\delta) \Gamma\left(u_{\ell}\right)} \\
& \times\left(2 K \frac{\left(T_{\ell}-T_{\ell-1}\right)^{u_{\ell}}}{\Gamma\left(u_{\ell}+1\right)}\left\|z_{\ell}-\widetilde{x}_{\ell}\right\|_{E_{\ell}}+L\left\|z_{\ell}-\tilde{x}_{\ell}\right\|_{E_{\ell}}\right) \\
& \leq \epsilon \frac{\left(T_{\ell}-T_{\ell-1}\right)^{u_{\ell}}}{\Gamma\left(u_{\ell}+1\right)}+\frac{2\left(T_{\ell}-T_{\ell-1}\right)^{u_{\ell}-1}\left(T_{\ell}^{1-\delta}-T_{\ell-1}^{1-\delta}\right)}{(1-\delta) \Gamma\left(u_{\ell}\right)} \\
& \times\left(2 K \frac{\left(T_{\ell}-T_{\ell-1}\right)^{u_{\ell}}}{\Gamma\left(u_{\ell}+1\right)}+L\right)\left\|z_{\ell}-\tilde{x}_{\ell}\right\|_{E_{\ell}} \\
& \leq \epsilon \frac{\left(T_{\ell}-T_{\ell-1}\right)^{u_{\ell}}}{\Gamma\left(u_{\ell}+1\right)}+\mu\|z-x\|,
\end{aligned}
$$

where

$$
\mu=\max _{\ell=1,2, \ldots, n} \frac{2\left(T_{\ell}-T_{\ell-1}\right)^{u_{\ell}-1}\left(T_{\ell}^{1-\delta}-T_{\ell-1}^{1-\delta}\right)}{(1-\delta) \Gamma\left(u_{\ell}\right)}\left(2 K \frac{\left(T_{\ell}-T_{\ell-1}\right)^{u_{\ell}}}{\Gamma\left(u_{\ell}+1\right)}+L\right) .
$$

Then

$$
\|z-x\|(1-\mu) \leq \frac{\left(T_{\ell}-T_{\ell-1}\right)_{\ell}}{\Gamma\left(u_{\ell}+1\right)} \epsilon .
$$


We obtain, for each $t \in J_{\ell}$,

$$
|z(t)-x(t)| \leq\|z-x\| \leq \frac{\left(T_{\ell}-T_{\ell-1}\right)^{u_{\ell}}}{(1-\mu) \Gamma\left(u_{\ell}+1\right)} \epsilon:=c_{f_{1}} \epsilon .
$$

Therefore, the BVP (1) is $(U H)$ stable.

\section{Example}

Let us consider the following fractional boundary value problem:

$$
\left\{\begin{array}{l}
{ }^{c} D_{0^{+}}^{u(t)} x(t)=\frac{t^{-\frac{1}{3}} e^{-t}}{\left(e^{\frac{t^{2}}{1+t}}+4 e^{2 t}+1\right)\left(1+|x(t)|+\left.\right|^{c} D_{0^{+}}^{u(t)} x(t) \mid\right)}, \quad t \in J:=[0,2], \\
x(0)=0, \quad x(2)=0 .
\end{array}\right.
$$

Let

$$
\begin{aligned}
& f_{1}(t, y, z)=\frac{t^{-\frac{1}{3}} e^{-t}}{\left(e^{e^{\frac{t^{2}}{1+t}}}+4 e^{2 t}+1\right)(1+y+z)}, \quad(t, y, z) \in[0,2] \times[0,+\infty) \times[0,+\infty) . \\
& u(t)= \begin{cases}\frac{3}{2}, & t \in J_{1}:=[0,1], \\
\frac{9}{5}, & \left.\left.t \in J_{2}:=\right] 1,2\right] .\end{cases}
\end{aligned}
$$

Then we have

$$
\begin{aligned}
& t^{\frac{1}{3}}\left|f_{1}\left(t, y_{1}, z_{1}\right)-f_{1}\left(t, y_{2}, z_{2}\right)\right| \\
& \quad=\left|\frac{e^{-t}}{\left(e^{e^{\frac{t^{2}}{1+t}}}+4 e^{2 t}+1\right)}\left(\frac{1}{1+y_{1}+z_{1}}-\frac{1}{1+y_{2}+z_{2}}\right)\right| \\
& \quad \leq \frac{e^{-t}\left(\left|y_{1}-y_{2}\right|+\left|z_{1}-z_{2}\right|\right)}{\left(e^{e^{\frac{2^{2}}{1+t}}}+4 e^{2 t}+1\right)\left(1+y_{1}+z_{1}\right)\left(1+y_{2}+z_{2}\right)} \\
& \quad \leq \frac{e^{-t}}{\left(e^{\frac{t^{2}}{1+t}}+4 e^{2 t}+1\right)}\left(\left|y_{1}-y_{2}\right|+\left|z_{1}-z_{2}\right|\right) \\
& \quad \leq \frac{1}{(e+5)}\left|y_{1}-y_{2}\right|+\frac{1}{(e+5)}\left|z_{1}-z_{2}\right| .
\end{aligned}
$$

Hence the condition (H2) holds with $\delta=\frac{1}{3}$ and $K=L=\frac{1}{e+5}$.

By (15), according to (7) we consider two auxiliary BVPs for Caputo fractional differential equations of constant order,

$$
\left\{\begin{array}{l}
{ }^{c} D_{0^{+}}^{\frac{3}{2}} x(t)=\frac{t^{-\frac{1}{3}} e^{-t}}{\left(e^{e^{\frac{1}{1+t}}}+4 e^{2 t}+1\right)\left(1+|x(t)|+\left.\right|^{c} D^{\frac{3}{2}} x(t) \mid\right)}, \quad t \in J_{1}, \\
x(0)=0, \quad x(1)=0
\end{array}\right.
$$

and

$$
\left\{\begin{array}{l}
{ }^{c} D_{1^{+}}^{\frac{9}{5}} x(t)=\frac{t^{-\frac{1}{3}} e^{-t}}{\left(e^{e^{\frac{t^{1}}{1+t}}}+4 e^{2 t}+1\right)\left(1+|x(t)|+\left.\right|^{c} D^{\frac{9}{5}} x(t) \mid\right)}, \quad t \in J_{2}, \\
x(1)=0, \quad x(2)=0 .
\end{array}\right.
$$


Next, we prove that the condition (9) is fulfilled for $\ell=1$. Indeed,

$$
\begin{aligned}
\frac{2\left(T_{1}^{1-\delta}-T_{0}^{1-\delta}\right)\left(T_{1}-T_{0}\right)^{u_{1}-1}}{(1-\delta) \Gamma\left(u_{1}\right)}\left(\frac{2 K\left(T_{1}-T_{0}\right)^{u_{1}}}{\Gamma\left(u_{1}+1\right)}+L\right) & =\frac{1}{\frac{2}{3}(e+5) \Gamma\left(\frac{3}{2}\right)}\left(\frac{2}{\Gamma\left(\frac{5}{2}\right)}+1\right) \\
& \simeq 0.3664<1 .
\end{aligned}
$$

Accordingly the condition (9) is achieved. By Theorem 3.1, the problem (16) has a solution $\tilde{x}_{1} \in E_{1}$.

We prove that the condition (9) is fulfilled for $\ell=2$. Indeed,

$$
\begin{aligned}
\frac{2\left(T_{2}^{1-\delta}-T_{1}^{1-\delta}\right)\left(T_{2}-T_{1}\right)^{u_{2}-1}}{(1-\delta) \Gamma\left(u_{2}\right)}\left(\frac{2 K\left(T_{2}-T_{1}\right)^{u_{2}}}{\Gamma\left(u_{2}+1\right)}+L\right) & =\frac{2^{\frac{2}{3}}-1}{\frac{2}{3} \Gamma\left(\frac{9}{5}\right)} \frac{1}{e+5}\left(\frac{2}{\Gamma\left(\frac{14}{5}\right)}+1\right) \\
& \simeq 0.2682<1 .
\end{aligned}
$$

Thus, the condition (9) is satisfied.

According to Theorem 3.1, the BVP (17) possesses a solution $\widetilde{x}_{2} \in E_{2}$.

Then, by Theorem 3.2, the BVP (14) has a solution

$$
x(t)= \begin{cases}\widetilde{x}_{1}(t), & t \in J_{1}, \\ x_{2}(t), & t \in J_{2},\end{cases}
$$

where

$$
x_{2}(t)= \begin{cases}0, & t \in J_{1}, \\ \widetilde{x}_{2}(t), & t \in J_{2} .\end{cases}
$$

According to Theorem 4.1, BVP (14) is $(U H)$ stable.

\section{Acknowledgements}

The third author would like to thank the Department of Mathematics, Faculty of Applied Science, King Mongkut's University of Technology North Bangkok.

\section{Funding}

This research was funded by Thailand Science Research and Innovation Fund, and King Mongkut's University of Technology North Bangkok with Contract no. KMUTNB-BasicR-64-33-1.

Availability of data and materials

Not applicable.

\section{Competing interests}

The authors declare that they have no competing interests.

\section{Authors' contributions}

All authors contributed equally to the writing of this paper. All authors read and approved the final manuscript.

\section{Author details}

${ }^{1}$ Laboratory ACEDP, University of Djillali Liabes, Sidi Bel Abbès, Algeria. ${ }^{2}$ Department of Economic Sciences, University of Tiaret, Tiaret, Algeria. ${ }^{3}$ Intelligent and Nonlinear Dynamic Innovations Research Center, Department of Mathematics, Faculty of Applied Science, King Mongkut's University of Technology North Bangkok (KMUTNB), Wongsawang, Bangsue, 10800, Bangkok, Thailand.

\section{Publisher's Note}

Springer Nature remains neutral with regard to jurisdictional claims in published maps and institutional affiliations. 


\section{References}

1. Benchohra, M., Lazreg, J.E.: Existence and Ulam stability for nonlinear implicit fractional differential equations with Hadamard derivative. Stud. Univ. Babeş-Bolyai, Math. 62(1), 27-38 (2017)

2. Benchohra, M., Lazrega, J.E.: Existence and uniqueness results for nonlinear implicit fractional differential equations with boundary conditions. Rom. J. Math. Comput. Sci. 4(1), 60-72 (2014)

3. da Vanterler, J., Sousa, C., Capelas de Oliverira, E.: Two new fractional derivatives of variable order with non-singular kernel and fractional differential equation. Comput. Appl. Math. 37, 5375-5394 (2018)

4. Gómez-Aguilar, J.F.: Analytical and numerical solutions of nonlinear alcoholism model via variable-order fractional differential equations. Physica A 494, 52-57 (2018)

5. Jiahui, A., Pengyu, C.: Uniqueness of solutions to initial value problem of fractional differential equations of variable-order. Dyn. Syst. Appl. 28(3), 607-623 (2019)

6. Kilbas, A.A., Srivastava, H.M., Trujillo, J.J.: Theory and Applications of Fractional Differential Equations. North-Holland Mathematics Studies, vol. 204. Elsevier Science B.V., Amsterdam (2006)

7. Samko, S.G.: Fractional integration and differentiation of variable order. Anal. Math. 21, 213-236 (1995)

8. Samko, S.G., Boss, B.: Integration and differentiation to a variable fractional order. Integral Transforms Spec. Funct. 1 , 277-300 (1993)

9. Sun, H., Chen, W., Wei, H., Chen, Y.: A comparative study of constant-order and variable-order fractional models in characterizing memory property of systems. Eur. Phys. J. Spec. Top. 193, 185-192 (2011)

10. Tavares, D., Almeida, R., Torres, D.F.M.: Caputo derivatives of fractional variable order numerical approximations. Commun. Nonlinear Sci. Numer. Simul. 35, 69-87 (2016)

11. Valerio, D., Costa, J.S.: Variable-order fractional derivatives and their numerical approximations. Signal Process. 91, 470-483 (2011)

12. Yang, J., Yao, H., Wu, B.: An efficient numerical method for variable order fractional functional differential equation Appl. Math. Lett. 76, 221-226 (2018)

13. Zhang, S.: Existence of solutions for two point boundary value problems with singular differential equations of variable order. Electron. J. Differ. Equ. 245, 1 (2013)

14. Zhang, S.: The uniqueness result of solutions to initial value problems of differential equations of variable-order. Rev. R. Acad. Cienc. Exactas Fís. Nat., Ser. A Mat. 112, 407-423 (2018)

15. Zhang, S., Hu, L.: Unique existence result of approximate solution to initial value problem for fractional differentia equation of variable order involving the derivative arguments on the half-axis. Mathematics 7(286), 1-23 (2019)

16. Zhang, S., Hu, L.: The existence and uniqueness result of solutions to initial value problems of nonlinear diffusion equations involving with the conformable variable. Azerb. J. Math. 9(1), 22-45 (2019)

17. Zhang, S., Hu, L.: The existence of solutions and generalized Lyapunov-type inequalities to boundary value problems of differential equations of variable order. AlMS Math. 5(4), 2923-2943 (2020)

18. Zhang, S., Sun, S., Hu, L:: Approximate solutions to initial value problem for differential equation of variable order. J. Fract. Calc. Appl. 9(2), 93-112 (2018)

\section{Submit your manuscript to a SpringerOpen ${ }^{\circ}$ journal and benefit from:}

- Convenient online submission

- Rigorous peer review

- Open access: articles freely available online

- High visibility within the field

Retaining the copyright to your article

Submit your next manuscript at $\gg$ springeropen.com 